\title{
Change of Soil Bacteria Diversity between Desertification and Restoration
}

\author{
Feijie $\mathrm{Li}^{1,2,3}$, Haiyan Jia ${ }^{1,2}$, Yan Wang ${ }^{1,2}$, Hui Tang ${ }^{1,2}$,Liping Zhang ${ }^{1,2,3 *}$ \\ ${ }^{1}$ (College of Life Sciences, Hebei University, Baoding 071002, PR China) \\ ${ }^{2}$ (The Key Lab of Microbial Diversity Research and Application of Hebei Province, Baoding 071002, PR China) \\ ${ }^{3}$ (Key Laboratory of Medicinal Chemistry and Molecular Diagnosis, Hebei UniversityMinistry of Education,
} Baoding 071002, PR China)

\begin{abstract}
The control of desertification has focused for centuries on plants and animals; microbial profiles are largely unknown. We collected soil samples from the aeoliansandy land (SL), sandy grassland (SG), and restored sandy land (RS), respectively. Diversity indexes were determined based on $16 S$ rDNA sequencing. Results show that $S L$ is of the highest species diversity and richness as deduced from Shannon index $(S L>R S>S G)$ and Menhinick index $(S L>R S>S G)$, while the microbial distribution is most homogeneous in $R S$ and most inhomogeneous in $S G$ as deduced from Evenness index (RS $>S L>S G)$. Acidobacteria, Proteobacteria, Actinobacteriaand Firmicuteswere among the highly abundant taxonomic groups present across the three sample sites and were designated as the core phyla. Acidobacteriawere dominant in SG(49.8\%) and RS (32.4\%), while Actinobacteria (23.9\%) and Firmicutes (22.6\%) were dominant in SL. Although the overall microbial composition was more similar between $S G$ and RS, there are more common genus shared by $S L$ and RS. Besides, compared with SL, only Acidobacteriaand Proteobacteriaincreased, Firmicutesand Actinobacteriadecreased greatly in $S G$ and RS. We try to explain the microorganisms' function in the progress of desertification and restoration, and to provide a theoretical basis for the recovery of aeolian sandy land.
\end{abstract}

Keywords: bacteria diversity, desertification, restoration, soil samples, $16 \mathrm{~S}$ rDNA

\section{Introduction}

Desertification was initially defined as the change of productive lands into desert, caused by human activity[1]. In 1977, the United Nations Environment Program defined desertification as "the diminution or destruction of biological potential of land which can lead ultimately to desert-like conditions"[2]. UN member countries have ratified the UNCCD (United Nations Convention to Combat Desertification) which provides international guidelines for responding to desertification. According to the Convention, desertification is "land degradation in arid, semi-arid and dry sub-humid areas resulting from various factors including climatic variations and human activities"[3].

The desert is a xeric environment, and is characterized by low and unpredictable rainfall amounts and frequency, low amounts of nutrients, and organic matter[4], [5], and temporal patterns of resource distribution. In these arid ecosystems, water availability and organic matter are the major factors limiting soil-biotic activity[6]. At present, the potential, mild and moderate desertification land occupies $60 \%$ of the total desertification land[7], [8]. But as long as human manage it properly, the desertification land is likely to improve and restore in a short period of time[9]. There has been a lot of papers reported that in arid and semi-arid areas, desertified conditions may allow for vegetation and landscape recovery at least temporarily[10]. And over periods of several decades, changes in rainfall regimes or human activity may allow for self-remediation or 'greening' of areas previously described as desertified[11]-[13].

Inner Mongolia, China has a vast territory and a variety of soil types (Fig. 1a[14]). Among those soil zones, aeolian sandy soil is widely distributed, and it can be found almost all the continents in the world[15], [16]. Aeolian sandy soil has some common characters compared with desert, but its development and utilization potential is larger, as long as treat it properly, it is possible for the recovery of aeolian sandy land. However, for a long time, people have not realized the value of aeolian sandy land. In recent years, with the control and management of the aeolian sandy land, the situation has been improved obviously[17]. In September and October 2012, we investigated the status of desertification in Plain Blue Banner, and sampled at the desertificated aeolian sandy land, restored aeolian sandy land and have not been desertificated aeolian sandy grassland. We constructed three $16 \mathrm{~S}$ rDNA clone libraries using the soil samples. Our purpose was just to study the microbial community and diversity, and the possible functions in desertification and restoration.

In soil ecological systems, soil microbes are predominant over animals and plants, and they are directly or indirectly involved in the diverse functions of the soil environment[18], playing key roles in energy flow, nutrient cycle, system stability, and carbon sequestration[19]-[23]. Microbial community is composed of a plurality of microbial population, its community structure and diversity can sensitively reflect the ecological 
functions and the environment changes, and it is a pioneer of the ecological environment restoration[24]. Largescale surveys conducted in recent years have shown that different ecosystems support unique microbial populations[25],[26]. A number of molecular microbial diversity studies of soil communities have been reported[27]-[30], but there has been no comparable analysis of the relationship between sandy land and the restored sandy land. In our attempt to study the microbial community composition within each of the sample sites we relied on a previous study[31] in which they tested bacterial diversity, using a scheme that enabled us to examine triplicate composite soil samples in each plot within each sample site. In their study, statistical analysis of bacterial fingerprints revealed that the differences among plots within each site are not statistically significant $(\mathrm{P}=0.33)$, indicating that the samples taken from the plots within a site are indeed replicates[32]. According to this, we decided to study the soil microbial community at a single representative plot within each sample site.

For most terrestrial ecosystems, soil microbial community structure has turned out to be strongly influenced by soil temperature, soil moisture, soil $\mathrm{pH}$, or a combination of these factors[26], [33]. Changes in the composition of the microbial community can subsequently affect the rates of decomposition and nutrient cycling dynamics[34], [35]. Studies have shown that water availability is one of themain limiting factors that largely determine community functions in sandy ecosystems. For many years, the ecological paradigm has been that water availability is positively correlated with richness, diversity and abundance of communities[26], [36]. While in recent years, it has been reported that the rules apply to microorganisms do not necessarily hold for microorganisms, and water availability is not directly correlated with the diversity of all organisms[26]. A broad scale survey aiming at finding robust patterns in the structure of soil microbial showed that unlike plant communities, which typically harbor distinct ecosystems, bacterial communities are rather similar in composition (harboring the same dominant phyla), while differ in structure and biomass[37]. Soil pH and available carbon could only explain the spatial variance of some bacterial phyla (Acidobacteria, $\beta$ Proteobacteria, and Bacteroidetes), while the variance of Actinobacteria, $\alpha$-Proteobacteriaand Firmicutescould not be assigned to the soil characteristics[38].

In our study, we focused on three relatively dry environments (sandy land, sandy grassland and restored sandy land), suggested that although bacterial abundance may relate to the water content, while bacterial richness and diversity may not. Each site supported a unique array of OTUs, which had little in common with the others. These observationssupport the hypothesis that spatial isolation of microbial populations in soils is a key determinant of microbial community structure[39]. Community composition can be correlated to an array of environmental factors. It has been suggested that different chemical components can alter microbial richness in soils. Yet it remains to be seen which of the detected correlations will prove to be significant to phylum diversity and composition[29].

\subsection{Field sites and conditions}

\section{Materials And Methods}

Plain BlueBanner is located in southern the Inner Mongolia Autonomous Region, China, $115^{\circ} 00^{\prime}-$ $116^{\circ} 42^{\prime} \mathrm{E}, 41^{\circ} 56^{\prime}-43^{\circ} 11^{\prime} \mathrm{N}$. Plain Blue Banner is located at the northern foot of Yinshan Mountains, composed of low mountains and hills and Hunshandake sandy land two majorlandforms, the general topography is characterized by the high East and low West, with an altitude of 1200-1600m. Located in the northern part of Hunshandake sandy land is mainly the aeolian sandy grassland, accounting for $66 \%$ of the total area of the whole banner; southern is low hill, mainly the typical steppe and meadow steppe, accounted for $34 \%$ of the total banner. The banner is semi-arid temperate continental monsoon climate, sandstorm, drought little rain, grassland degradation, desertification, frequent natural disasters are the main characteristics. The aeolian sandy grassland desertification degree can be divided into four categories, non desertification sandy grassland (vegetation coverage $\geq 60 \%$ ), mild desertification sandy grassland (vegetation coverage $30 \%-60 \%$ ), moderate desertification sandy grassland (vegetation coverage 10\%-30\%) and severe desertification sandy grassland (vegetation coverage $<10 \%$ ). In our study, we choose the non desertification sandy land (sandy grassland), the severe desertification sandy land (sandy land) and restored desertification sandy land (restored sandy land) as our sample sites. The selection of sampling sites was based on the vegetation coverage change in Plain Blue Banner during 2000-2009 (Fig. 1b). In order to facilitate the description we named the sandy grassland as SG, the sandy land as SL and the restored sandy land as RS.

\subsection{Soil sampling}

The soil sample (depth 5-20cm) was collected from SL, SG and RS in September and October 2012 (Fig. 1b). Sampling at each site was based on a spatially stratified, random sampling approach: at each site each of the three plots was divided into equal sectors of $1 \mathrm{~m} 2$, and for each plot, fresh soil samples were collected randomly from nine points. In the laboratory, the nine soilsamples of each plot were mixed and sieved $(<2 \mathrm{~mm})$, and then conserved at $-70^{\circ} \mathrm{C}$ for the next study. Soil samples from three replicate plots of each treatment were mixed for DNA extraction. 


\subsection{Screening of a 16S rDNA clone library for matching sequences}

Total soil DNA was extracted using a SoilMasterTM DNA Extraction Kit (Epicentre, Madison, WI), and then purified using the Soil DNA Purification Kit (Genmed Scientifics Inc.). 16S rDNA sequences were amplified with a GeneAmp PCR System 2400 thermocycler, using 30 cycles of $94^{\circ} \mathrm{C}$ for $30 \mathrm{~s}, 56^{\circ} \mathrm{C}$ for $30 \mathrm{~s}$, and $72^{\circ} \mathrm{C}$ for $1 \mathrm{~min}$. The PCR mixtures $(50 \mu \mathrm{L})$ contained $10 \mathrm{mM}$ Tris- $\mathrm{HCl}(\mathrm{pH} 8.3), 50 \mathrm{mM} \mathrm{KCl}, 3 \mathrm{mM} \mathrm{MgCl} 2$, $150 \mathrm{mM}$ dNTP, 30 pmol of primers $27 \mathrm{~F}$ and 1392R (Escherichia coli $16 \mathrm{~S}$ rDNA sequence numbering), 2.5U of Taq DNA polymerase (Takara Bio Group), and $1 \mu \mathrm{L}$ of template DNA (approximately 100ng). To recondition the PCR product for elimination of heteroduplexes in mixed-template PCR[40], the amplified reaction was diluted 10-fold into a fresh reaction mixture of the same composition and cycledthree times using the parameters specified above. The size and quality of the resulting PCR products was confirmed by agarose gel electrophoresis (1.4\% agarose). They were then cloned into the pUCm-T linear plasmid vector (Takara Bio Group, Code D101A) and then into E. coliDH5 $\alpha$ competent cells as specified by the manufacturer (Takara Bio Group). After the transformants were grown overnight, single-clone colonies were picked up with sterile toothpicks and transferred into $1.5-\mathrm{mL}$ microcentrifuge tubes containing $50 \mu \mathrm{L}$ of TE buffer. The tubes were heated for $15 \mathrm{~min}$ at $95^{\circ} \mathrm{C}$ to lyse the cells, and then chilled on ice. Insert sequences were amplified with a thermocycler (as above), using 25 cycles of $94^{\circ} \mathrm{C}$ for $30 \mathrm{~s}, 46^{\circ} \mathrm{C}$ for $30 \mathrm{~s}$, and $72^{\circ} \mathrm{C}$ for $50 \mathrm{~s}$. The PCR mixtures $(20 \mu \mathrm{L})$ contained $10 \mathrm{mM}$ Tris- $\mathrm{HCl}(\mathrm{pH} 8.3), 50 \mathrm{mM} \mathrm{KCl}, 3 \mathrm{mM} \mathrm{MgCl} 2,150 \mathrm{mM}$ each dNTP, 3pmol of primers M13/pUC sequencing primer (-20) and M13/pUC reverse primer (-26), 1.0U of Taq DNA polymerase, and $1 \mu \mathrm{L}$ of cell lysate. The vector-specific primers -20 and -26 amplified the region between the multiple cloning sites where the amplicons should be inserted (approximately $1.5 \mathrm{~kb}$ ), identified by agarose gel electrophoresis, then sequenced by Shanghai Sangon. Sequences were compared with the GenBank database by BLAST searches[41]using the MEGABLAST option to identify the closest matches. All sequences were evaluated for chimeric sequences using the NAST sequence alignment and CHECK_CHIMERA tools[42].

\subsection{Data analysis}

Sequences were aligned using CLUSTAL X[43] and imported into DNADIST in PHYLIP version 3.6[44]to generate distance matrices using the Juke-Cantor correction for multiple substitutions, and then operational taxonomic units (OTUs) were assigned by DOTUR[45]. Unique phylotypes were defined as OTUs with $<99 \%$ 16S rDNA sequence similarity as determined by GCG BESTFIT software. To estimate species richness, the nonparametric Chao1 estimate was calculated with log-linear-transformed confidence intervals at 95\%[46]. Coverage (C) was used as a measurement of captured diversity, where $\mathrm{C}$ is expressed by $1-\mathrm{n} 1 / \mathrm{N}$, in which $n 1 / \mathrm{N}$ is the ratio of clones that appeared only once ( $\mathrm{n} 1)$ tothe total number of clones $(\mathrm{N})$ [47]. A number of unique phylotypes (phylotype richness, S) were used for the creation of rarefaction curves[48]. The PAST (http://folk.uio.no/ohammer/past) was used to calculate the Simpson index $=1-\sum(\mathrm{ni} / \mathrm{n}) 2$, which measures 'evenness' of the community from 0 to 1 (note the confusionin the literature), where ni is the number of individuals of taxon t. Evenness $=\mathrm{eH} / \mathrm{S}$, where $\mathrm{S}$ is the number of taxa. Menhinick's richness index, the ratio of the number of taxa to the square root of sample size. The original data that matrixed with phylotype in row and farming model in the columnwere Z-standardized, and then input to PAST to produce a var-covar matrix for principal component analysis (PCA). To exclude obvious chimeric 16S rDNA primary structures before the phylogenetic analysis[49], the 'CHECK_CHIMERA' program of the Ribosomal Database Project (RDP)[50]was used, and a separate treeing analysis of the terminal 400 nucleotide sequence positions at the 5' and 3' ends of the environmental $16 \mathrm{~S}$ rDNA clones was carried out. The overall levels of similarity between $16 \mathrm{~S}$ rDNA sequences were determined using the appropriate tool of the ARB program package. 16S rRNA sequences used the online alignments and analysis (http://rdp.cme.msu.edu/). In order to assess the phylogenetic distribution and taxonomic diversity of the soil samples, sequences were classified with the Ribosomal Database Project Classifier at 0.8 confidence threshold[8].

\subsection{Nucleotide sequence accession numbers}

All the sequences of the 16S rRNA gene clone libraries were deposited in GenBank with submissions number grp 5213155 .

\subsection{Microbial Diversity}

\section{Results}

We constructed threeclone libraries, to analyzethe composition and change of microbial flora. Ultimately, the clone libraries amplified from the SL, SG and RS yielded472, 359, and 414 high-quality clones. In order to compare the microbial diversity within the three samples, $16 \mathrm{~S}$ rDNA gene sequences of the isolates showing $\geq 97 \%$ sequence similarity were grouped into the same OTU, and at last yielded 174, 105and 126 OTUs0.03, respectively. A comparison of the OTUs0.03 resulted in the identification of 313 OTUs in total, with 24 common OTUs shared by the three sample sources. Fig. 2depicts a Venn diagram representing the unique 
and overlapping OTUs at the three sample sites, indicating that most of the members of the soil communities were unique to their environment.

Rarefaction curves (Fig. 3) andCoverage indicated that the bacteria identified from the community DNA extracts from the SL, SG and RS samples were sampled adequately, the number of sequenced clones from each library was sufficient tocover the mostenvironmental microbial diversity, and thus they were analyzed further for diversity characterization.

The microbial diversity appears to be differentamong the three sample sites, and SL is of the highest species diversity and richness as deduced from Shannon index(SL $>$ RS $>$ SG)and Menhinick index $(\mathrm{SL}>\mathrm{RS}>\mathrm{SG})$. The Evenness is RS $>\mathrm{SL}>\mathrm{SG}$, it means that the population quantities were distributed non-uniformly, and RS is tended to be more evenly distributed.From the indexes we can summarize that SL is of the highest species diversity, abundance and dominant species, while the species evenness is at the middle level compared with RS and SG; SG has the lowest species diversity, abundance and dominant species, besides, the species is more unevenly distributed than others; RS is at the middle level of species diversity, abundance and dominant species, while the species distribution is the most homogeneous.

\subsection{Phylogenetic structure of microbial communities}

The composition of microbial communities was determined by analysis of 16S rRNA gene clone libraries. A total of 613 partial sequences were obtained from the three clone library. These 16S rRNA sequences were compared with sequences from reference and type strains, as well as environmental clones, available at the GenBank and RDP II databases. Through the identification and classification of representative sequences in each OTUs0.03, clone sequences were attributed to 14 phyla, which were shared by the three sample sites.Fig. 4depicts the different bacterial phyla as number of all group members detected in each sampling site. We can note that in the three sample sites the overall phylum-level composition was similar and the Acidobacteria occupiedthe largest proportion, then followed by Proteobacteria, Firmicutes and Actinobacteria. The four phyla were the most predominant of the total clones. Verrucomicrobia, Chloroflexi, Gemmatiomonadetes, Bacteroidetes, Planctomycetes, Nitrospira, Deinococcus-Thermus and Armatimonadetes were present in one or two libraries but the frequency of distribution was truncated. These observations are in accord with some other earlier studies[51]-[53].

There are great differences in phyla abundance between different soil samples. Fig. 5 showed the percentage of each phylum in the three soil samples. In SL, it was dominated by Actinobacteria(23.94\%), then followed by Firmicutes $(22.67 \%$ ) and Proteobacteria(17.16\%). In SG, the most abundant phylum is Acidobacteria, accounting for almost 49.30\%, then followed by Proteobacteria $(21.29 \%)$ and Firmicutes(14.01\%). In RS, the Acidobacteria is also the most abundant, but the proportion has been reduced to $32.37 \%$, then followed by Proteobacteria $(22.46 \%)$ and Firmicutes $(18.36 \%)$. In phylum Proteobacteria, there are four classes, Alpha-, Beta-,Delta-and Gammaproteobacteria, from the figure we can see that the percentage of Alphaproteobacteria is almost the same in three sample sites, while Betaproteobacteria is highest in SG and nearly twice the percentage than in SL and RS. The percentage of Deta-and Gammaproteobacteria is most high in RS, then is SL and SG.

\section{Discussion}

The formation of aeolian sandy soil can be roughly divided into three stages: aeolian sandy soil stage (Sandy parent material contains a certain amount of nutrients and moisture, provides conditions for sand vanguard plant to grow, but because of the wind erosion and mobile, growth is very sparse, and coverage is less than 10\%), semi fixed aeolian sandy soil stage (plant coverage often between 10\% 30\%, wind erosion weakened,and showed some characteristics of soil formation), and fixed aeolian sandy soil stage (plant coverage is more than $30 \%$, in additionto the sand plant, there are some zonality plant components, raw soil pedogenesis is significantly). With the development of fixed aeolian sandy soil it may form the corresponding zonal soil.

In the process of aeolian sandy soil reserved to fixed aeolian sandy soil, environment,pioneer plant and microorganisms may play important roles. Soil microorganism has a broad ecological adaptation to the desert area of extreme environment, a lot of drought or high temperature resistant microbial species can change the sand surface physicochemical properties through the physiological and metabolic activities, and play a positive role inthe formation of soil and the process of plant nutrition transformation. Microorganism is conductive to the growth of plants, and thus lay the foundation of transforming moving dune to fixed or semi fixed dune. At the same time, the composition, quantity and distribution of soil microorganism can be influenced by the physical and chemical properties of soil, sand dune fixation duration, soil layer, sand layer depth, season, vegetation and other factors, thus showing a certain amount, composition and distribution change regulation.Our purpose is to find the microorganisms which may play a critical role in the restoration of sandy land, and thus can provide some theoretical basis for the comprehensive treatment of sandy land.

\subsection{The Phylum Level Analysis}


From these data showed above we can find that the distribution of different kinds of microbes in the three sample plots is of major difference. Acidobacteria, Actinobacteria, Proteobacteria and Firmicutes all emerged in the three plots, and occupied a large proportion, except them, Chloroflexi, Gemmatimonadetes and Nitrospira also found in those plots. Although the seven biological groups shared by the three sample, the gap in their number is great. Phyla present in the SL and RS sample but absent in the SG sample including Bacterdidetes, Deinococcus-Thermus, Nitrospira and Verrucomicrobia. Only one phylum Armatimonadetes was shared by SL and SG. No phylum was found only shared by SG and RS. BRC1 was only found in SG.

Acidobacteria is a newly separated phylum,and is categorized as acidophilic bacteria. Acidobacteria represents abundant members of soil microbial communities but only few representatives could be isolated and validly described so far[54]. Culture-independent studies indicate that the diversity ofthe phylum Acidobacteria is nearly as great as the diversity of the phylum Proteobacteria[55], and currently comprise 26 phylo-genetically distinct subdivisions[56]. In 16S rRNA gene libraries, they constitute an average fraction of $20 \%$ that are only surpassed by Proteobacteria[57]. The Acidobacteria is one of the most common bacteria in the soil samples, prefer low $\mathrm{pH}(3.5 \sim 4.5)$ and use glucose as one of the most favorable substrates[58]. Acidobacteria plays an important role in the ecosystem, especially in soil. There is a significant increase in SG and RS compared with $\mathrm{SL}$, the reason maybe that the soil pH in SG and RS is lower than SL and thus provide a suitable environment for the Acidobacteria to grow. Soil pH is affected by many factors, such as the climate, topography, soil parent material, vegetation, etc. The vegetation effect on soil $\mathrm{pH}$ is mainly due to plant roots had selective absorption of ions. In addition, soil microbial activity also has a great effect on soil $\mathrm{pH}$, because some kind of microbecan decompose organic or inorganic compounds in soil, and microorganism itself may secreted some substances, thus causing the change in soil $\mathrm{pH}$.

Actinobacteria is knew for its ability of surviving under adverse conditions. Actinobacteriaall believed to have high guanine and cytosine content in their DNA[59], and it is one of the dominant bacterial phyla and contains one of the largest bacterial genera, Streptomyces[60]. Actinobacteria plays an important role in the decomposition of organic matter and the antagonism of plant pathogens, thus, a reduction of their group and proportion in soil may lead to an increase in the number of pathogens, as well as a reduction of effective nutrients. And the phylum abundance was positively correlated with nitrate and magnesium, but negatively correlated with phosphorus. In general, Actinobacteria is especially abundant in the soil which is relatively dry, neutral to alkaline and containing a number of organic substances. However, compared with SG and RS, our results revealed an outstanding high abundance in SL. This is likely because although SL soil organic matter content is not very high, but SL have a low water content, and the soil $\mathrm{pH}$ is more close to neutral and alkaline, thus resulting in the high content in SL.

Change regulation of Firmicutes in the three sample sites is almost the same in comparison with Actionbacteria. According to Fierer N, the phylum Firmicutesis correlated negatively with soil moisture[38], and was abundant in the deserts[61], [62]. And most of the Firmicutes are capable of forming droughtwithstanding spores, this makes them able to survive in arid and hot environments. Besides, the research[57], [63]indicate that SL can lead to the loss of soil moisture. These may be the reasons that Firmicutes significantly more abundant in SL than in SG and RS.

All Proteobacteria are Gram-negative, with an outer membrane mainly composed of lipopolysaccharides. Proteobacteria can divide into six sections, including Alpha-, Beta-, Gamma-, Delta-, Epsilon-, andAcidithiobacillia. They include a wide variety of pathogens, such as Escherichia, Helicobacter, Salmonella, Vibrio, and many other notable genera. Others are free-living (non-parasitic) bacteria, including many of the bacteria which are responsible for nitrogen fixation. There is also a wide variety type ofmetabolism. Most of them are facultatively or obligatelyanaerobic, chemoautotrophs, and heterotrophic, but there are also exceptions, such as purple bacteria. From Fig. 3 we can see that Proteobacteria were not significantly changed in the three sites, although an increasing trend was detected in the RS sample in comparison with SG and SL. In the three samples, we only found four classes (Alpha-, Beta-, Gamma-and Deltaproteobacteria), but there is a large difference between the four classes shared in each sample.

Other phyla Chloroflexi, Bacteroidetes, Gemmatimonadetes, Planctomycetes, verrucomicrobia, Nitrospira, Deinococcus-Thermus, BRC1 and Armatimonadetes were not significantly abundant in any of the sample sites, and Bacteroidetes, verrucomicrobia, Nitrospira and Deinococcus-Thermus only find in SL and RS. This is likely due to RS is the result of partial recovery of SL, and there is still a certain gap compared with SG, though RS and SG are all sandy grassland, thus it is normal for some microbial species only exist in SL and RS. In addition, SG ecological system has stabilized to some extent, the composition of microbial communities tend to be relatively fixed, while SL and RS are still in the development and change stage of the environment, microbial composition has to change to adapt to the continuous development of environment and soil conditions.

In spite of the great bacterial diversity frequently found in soils, it is possible to recognize a certain pattern regarding the contribution of each phylum. The great majority of clones are usually affiliated to nine 
major bacterial phyla and generating stability in the community structure to a certain extent. According to Janssen[57], dominant phyla usually correspond to approximately $92 \%$ of the libraries, being Acidobacteria and Proteobacteria the most abundant ones, while Actinobacteria would only occupy $13 \%$ of total clones. In our study, phylogenetic analysis of the bacterial community of the threesample sites showed a different distribution compared with the general pattern. In SL, Actinobacteria and Firmicutes occupied nearly half of the library, while Proteobacteria and Acidobacteria represented only $17.6 \%$ and $13.1 \%$, respectively. In SG, Acidobacteria had a contribution of almost half of the library, Proteobacteria, Firmicutes and Actinobacteria represented only $21.6 \%, 14.0 \%$ and $0.8 \%$, respectively. In RS, Acidobacteria and Proteobacteria occupied more than half of the library, while Firmicutes and Actinobacteria represented only $17.1 \%$ and $6.5 \%$, respectively.

\subsection{The microbial change regulation}

Table 2 showed the comparison of microorganisms in each library at the phylum, class and order level. From the figure we can roughly compare the changes between three sample sites, here we compared the changes from SG to SL and from SL to RS, our purpose is to find the possible key microbial groups in the change of soil sample sites from sandy grassland to sandy land and from sandy land to restored sandy land.

Changes from SG to SL. From table 2 we can easily find Acidobacteria has a great decrease in the percentage of each library(from $49.3 \%$ to $12.9 \%$ ), and a great increase in Actinobacteria (from $0.8 \%$ to $23.9 \%$ ). In Actinobacteria, we only find one class Actinobacteria, and the orders Acidimicrobiales, Actinomycetales and Solirubrobacterals increased greatly from SG to SL. The phylum Firmutes also has an increase in SL (from $14.0 \%$ to $22.7 \%$ ) compared with SG. But in Firmutes, only class Bacilli increased from $10.1 \%$ to $22.4 \%$, and the main orders are Bacillales (from $10.1 \%$ to $21.8 \%$ ) and Lactobacillales (from 0 to $0.6 \%$ ), while the class Clostridia dropped greatly from $3.9 \%$ to $0.2 \%$. In phylum Proteobacteria, though the clone number increased in SL compared with SG, the percentage has decreased from $21.2 \%$ to $17.2 \%$, and among the four classes contained in this phylum, Alpha-and Betaproteobacteria decreased greatly, while Delta-and Gammaproteobacteria increased to some extent. In phylum Chloroflexi, there is an increasein SL compared with SG (from 1.4\% to 2.7\%), and three classes Anaerolineae, Caldilineales and Thermomicrobia only find in SL, and Chloroflexi only find in SG. There are four phyla increased slightly and only find in SL compared with SG, including Bacteroidetes, Deinococcus-Thermus, Nitrospira and Verrucomicrobia. Armatimonadetes and Gemmatimonadetes also increased in SL compared with SG (from $0.3 \%$ to $0.4 \%$ and $4.7 \%$ to $5.1 \%$, respectively), while Plactomycetes decreased from $0.6 \%$ to $0.2 \%$. Besides, BRC1 only find one clone and belonged to SG. Here we can see, in the change procession from SG to SL, except the percentage of phylum Acidobacteria, Plactomycetes and the class Clostridia decreased, others are all increased in some degree.

Changes from SL to RS. Compared the change tendency with SL to SG, the changes in SL to RS are almost the same in phylum Acidobacteria, Actinobacteria, Armatimonadetes, Chloroflexi, Firmicutes, Gemmatimonadetes,Plactomycetes, Verrucomicrobia and Proteobacteria, except the phylum Bacteroidetes, Deinococcus-Thermus and Nitrospira. In the last three phyla (Bacteroidetes, Deinococcus-Thermus and Nitrospira), there is an increasing tendency in RS compared with SL, which is opposite with the change from SL to SG. Besides, the last three phyla only exist in SL and RS, while absent in SG. Although the total change tendency is almost the same, there still exist some differences. In Proteobacteria, Delta-and Gammaproteobacteria increased from SL to RS, while decreased from SL to SG.

Comparingthe three sample sites together, the overall phylum-level composition was similar between SG and RS locations and dominated by Acidobacteria, followed by Proteobacteria, Firmicutes, and Actinobacteria. SL was dominated by Actinobacteria, followed by Firmicutes, Proteobacteria and Acidobacteria, which are exactly the backwards of the order of SG and RS.

Considering the data above, we can summarize that only Acidobacteria and Proteobacteria increased in SG and RS compared with SL, while Firmicutes and Actinobacteria decreased greatly in SG and RS. The four phyla may play the key role in soil development from SG degenerated to SL and SL restored to RS. And thus the increase of Acidobacteria and Proteobacteria and the decrease of Firmicutes and Actinobacteria may help sandy land restored to arable sandy grassland. Besides, we infer that microbes increased obviously in RS compared with SL and RS, or the microbes only exist in RS may play more important roles in the recovery of sandy land to sandy grassland. Here we find some microbes in order level may perform this function, including Rubrobacterales, Sphingobacteriales, Chloroflexales, Deinococcales, Selenomonadales, Nitrospirales, Phycisphaerales, Nitrosomonadales, Legionellales, Pseudomonadales and Verrucomicrobiales.

\subsection{The genus level analysis}

Results showed that, 613 sequences were identified to 123 different bacterial genera, and there are 73 genera contained in SL, 48 genera in SG, and 69 genera in RS. There are 17 genera shared by three sample sites, 21 genera shared by SL and RS, 6 genera shared by SL and SG, and 6 genera shared by SG and RS, besides, there are 29 genera only belonged to SL, 32 genera only belonged to SG and only 25 genera only belonged to RS. SL 
is of the highest microbial diversity, then followed by RS and SG. Though the clone number of common genus in SL and RS is not very high, there is an interesting phenomenon, that is compared with the common genera shared by SL and SG, as well as SG and RS, there are more genus shared by SL and RS. SL is the representative of aeolian sandy soil, while SG and RS are the representative of developed aeolian sandy soil, theoretically, due to the similarity of the environment, the microbial diversity in SG and RS should be more similar, however, the common genera are more shared in SL and RS, the reason may be that in the changing progress from SL to RS, part of the microbial species have good adaptability to environment, and result in part of the microorganisms in SL can still survive in RS. Besides, RSmay not fully restore to the stage as SG, and there may exist some transition types of microorganisms in RS, when RS developed further, the type and composition of microorganisms are likely to be closer to SG. And this phenomenon has already can be seen from the analysis before that comparing the three sample sites together, the overall phylum-level composition was more similar between SG and RS.

There are some genera only exist in RS, we guess that they may appear in the stage of SL recover to RS, while disappeared when RS totally recovered to the stage of SG. These genera may play an important role in the recovery of sandy land, including Bryobacter, Holophaga, Terriglobus, Amycolatopsis, Propionibacterium, Rubrobacter, Streptosporangium, Rhodocytophaga, Bellilinea, Blastopirellula, Gemmata, Verrucomicrobium, Exiguobacterium, Robinsoniella, Sporacetigenium, Staphylococcus, Devosia, Rhodoplanes, Delftia, Geoalkalibacter, Kofleria, Sorangium, Acinetobacter, Aquicella and Arenimonas. Besides the genera only belonged to RS and SG may also have this function, just including Gp7, Pedomicrobium, Rubellimicrobium and Massilia.

A meta-analysis of soil bacteria across seven of the major biomes on earth revealed that all biomes are dominated by the same soil bacterialphylum (Acidobacteria, Actinobacteria, Proteobacteria, and Firmicutes). Yet, although distinct biomes harbored similar bacterial communities, the composition of the bacterial communities varied and could be explained mostly by soil conditions. From Fig. 6 we can see that the first two variation can explain $100 \%$ of variance, and from the unifrac significance we can find that the SL and SG sample's environment is of marginally significant difference, but the SG and RS sample are not of great significance.

\section{Conclusion}

The aeoliansandy land(SL) hasthe highest species diversity and richness as deduced from Shannon indexand Menhinick index, while the microbial distribution is most homogeneous in restored sandy (RS) land and most inhomogeneous in sandy grassland (SG) as deduced from Evenness index. Acidobacteria,Proteobacteria, Actinobacteria and Firmicutes were among the highly abundant taxonomic groups present across the three sample sites and were designated as the core phyla. Acidobacteria were dominantin SG(49.8\%) and RS (32.4\%), while Actinobacteria (23.9\%) and Firmicutes (22.6\%) were dominant in SL. Although the overall microbial composition was more similar between SG and RS, there are more common genus shared by SL and RS. Besides, compared with SL, only Acidobacteria and Proteobacteria increased, Firmicutes and Actinobacteria decreased greatly in SG and RS. We try to explain the microorganisms' function in the progress of desertification and restoration, and to provide a theoretical basis for the recovery of aeolian sandy land.

\section{Acknowledgements}

The work was supported by the National Natural Science Foundation of China (No.30970010)and Bioengineering key disciplineof HebeiProvince.

\section{References}

[1]. S. M. Herrmann and C. F. Hutchinson, The changing contextsof the desertification debate,J. Arid Environ., 63(3), 2005,538-555.

[2]. R. Lal, Potential of Desertification Control to Sequester Carbon and Mitigate the Greenhouse Effect, Clim. Change,51(1),2001,3572.

[3]. N. S. F. S. D. London and N. Unep, United Nations Convention to Combat Desertification in Countries Experiencing Serious Drought and/or Desertification, particularly in Africa,1994.

[4]. E. Michael, S. Leslie, and T. Naphtali, The Negev: the challenge of a desert [Israel]. 2nd ed(the United States of America,Harvard University Press,1982).

[5]. W. G. Whitford and E. L. Wade, Ecology of desert systems,J. Mammal., 2002(3),1122.

[6]. I. Noy-Meir, Desert Ecosystems: Higher Trophic Levels, Ecol. Evol. Syst.,5(5), 1974,195-214.

[7]. W. Tao, Progress in sandy desertification research of China,J. Geogr. Sci.,14(4), 2004, 387-400.

[8]. Q. Wang, G. M. Garrity,J. M. Tiedje, and J. R. Cole, Naïve Bayesian Classifier for Rapid Assignment of rRNA Sequences into the New Bacterial Taxonomy,Appl. Environ. Microbiol., 73(16),2007, 5261-7.

[9]. T. H. Zhang et al., A comparison of different measures for stabilizing moving sand dunes in the Horqin Sandy Land of Inner Mongolia, China.,J. Arid Environ., 58(2), 2004, 203-214.

[10]. C. J. Tucker and W. W. Newcomb, Expansion and contraction of the sahara desert from 1980 to 1990.,Science, 253(5017), $1991,299-300$ 
[11]. C.F. Hutchinson, S.M. Herrmann, T. Maukonen, and J. Weber, Introduction: The 'Greening' of the Sahel,J. Arid Environ., 63(3),2005,535-537.

[12]. S. Nicholson, On the question of the 'recovery' of the rains in the West African Sahel.,J. Arid Environ.,63(3),2005,615-641.

[13]. L. Olsson, L. Eklundh, and J. Ardoe, A Recent Greening of the Sahel: Trends,Patterns and Potential Causes, J. Arid Environ., 63(3),2005,556-566.

[14]. M. A. Na, H. U. Yun-Feng, D. F. Zhuang, and X. L. Zhang, Vegetation Coverage Distribution and Its Changes in Plan Blue Banner Based on Remote Sensing Data and Dimidiate Pixel Model, Sci. Geogr. Sin., 32(2),2012, 251-156.

[15]. W. Liang,X. Wu, S. Zhang, and Y. Xing, Effect of organic amendments on soil water storage in the aeoliansandy land of northeast China, Chinese Academy of Sci, 2011,1538-1540.

[16]. H. C. Duan, T. Wang, X. Xue, S. L. Liu, and J. Guo, Dynamics of aeolian desertification and its driving forces in the Horqin Sandy Land, Northern China.,Environ. Monit. Assess., 186(10),2014,6083-96.

[17]. S. Liu and W. Tao, Aeolian desertification from the mid-1970s to 2005 in Otindag Sandy Land, Northern China, Environ. Geol., 51(6),2007,1057-1064.

[18]. J. Rombke and A. M. Breure, Status and outlook of ecological soil classification and assessment concepts,Ecotoxicol. Environ. Saf., 62(2),2005,300-308.

[19]. P. Nannipieri, J. Ascher, M. T. Ceccherini, L. Landi, G. Pietramellara, and G. Renella, Microbial diversity and soil functions, Eur. J. Soil Sci.,54(4),2003,655-670.

[20]. J. M. Lynch et al., Microbial diversity in soil: ecological theories, the contribution of molecular techniques and the impact of transgenic plants and transgenic microorganisms., Biol. Fertil. Soils, 40(6),2004,363-385.

[21]. C. E. Prescott, Do rates of litter decomposition tell us anything we really need to know?,For. Ecol. Manag., 220(1-3), 2005,66-74.

[22]. R. Angel, M. I. M. Soares, E. D. Ungar, and O. Gillor, Biogeography of soil archaea and bacteria along a steep precipitation gradient, Isme J.,4(4), 2010,553-63.

[23]. A. Bachar et al., Soil microbial abundance and diversity alonga low precipitation gradient., Microb. Ecol.,60(2), 2010,453-61.

[24]. D. A. Heemsbergen, M. P. Berg, M. Loreau, J. R. van Hal, J. H. Faber, and H. A. Verhoef, Biodiversity effects on soil processes explained by interspecific functional dissimilarity., Science, 306(5698), 2004,1019-20.

[25]. X. L. Wang, X. Zhang, Y. F. Zhou, and D. U. Yan-Long, An Analysis on Diversity of MicrobialCommunity Function in Desert,Bull. Soil Water Conserv.,32(3),2012,91-95.

[26]. N. Fierer and R. B. Jackson, The diversity and biogeography of soil bacterial communities.,Proc. Natl. Acad. Sci. U. S. A., 103(3), 626-31.

[27]. C. R. Kuske,S. M. Barns, and J. D. Busch, Diverse uncultivated bacterial groups from soils of the arid southwestern United States that are present in many geographic regions.,Appl. Environ. Microbiol., 63(9),1997,3614-21.

[28]. J. Borneman and E. W. Triplett, Molecular microbial diversity in soils from eastern Amazonia: evidence for unusual microorganisms and microbial population shifts associated with deforestation.,Appl. Environ. Microbiol.,63(7), 1997,2647-2653.

[29]. J. Zhou, B. Xia, H. Huang, A.V. Palumbo, and J. M. Tiedje, Microbial Diversity and Heterogeneity in Sandy Subsurface Soils, Appl. Environ. Microbiol., 70(3), 2004,1723-34.

[30]. O. C. Chan et al., 16S rRNA gene analyses of bacterial community structuresin the soils of evergreen broad-leaved forests in southwest China., FEMS Microbiol. Ecol.,58(2),2006, 247-259.

[31]. [31]W. Ludwig et al., Detection and in situ identification of representatives of a widely distributed new bacterial phylum, FEMS Microbiol. Lett.,153(1),1997,181-190.

[32]. [32]E. Stackebrandt,W. Liesack, and B. M. Goebel, Bacterial diversity in a soil sample from a subtropical Australian environment as determined by $16 \mathrm{~S}$ rDNA analysis., Faseb J.,7(1), 1993,232-236.

[33]. C. Bell, N. Mcintyre, S. Cox, D. Tissue, and J. Zak, Soil Microbial Responses to Temporal Variations of Moisture and Temperature ina Chihuahuan Desert Grassland,Microb. Ecol.,56(1),2008,153-167.

[34]. K. Kloos, U. M. Hüsgen, and H. Bothe, DNA-probing for genes coding for denitrification, N2, Z. Für Naturforschung C J. Biosci.,53(1-2), 1998,69-81.

[35]. R. I. Griffiths, A. S. Whiteley, A. G. O’Donnell, and M. J. Bailey, Influence of depth and sampling time on bacterial community structure in an upland grassland soil, FEMS Microbiol. Ecol.,43(1), 2003, 35-43.

[36]. B. A. Hawkins and J. R. G. Turner, Energy, Water, and Broad-Scale Geographic Patterns of Species Richness,Ecology, 84(12),3105-3117.

[37]. N. Fierer, M. S. Strickland, D. Liptzin, M. A. Bradford, and C. C. Cleveland,Global patterns in belowground communities., Ecol. Lett., 12(11),2009,1238-49.

[38]. N. Fierer, M. A. Bradford, and R. B. Jackson, TOWARD AN ECOLOGICAL CLASSIFICATION OF SOIL BACTERIA, Ecology,88(6), 2007, 1354-64

[39]. D. S. Treves, B. Xia, J. Zhou, and J. M. Tiedje, A two-species test of the hypothesis that spatial isolation influences microbial diversity in soil,Microb. Ecol., 45(1), 2003,20-28.

[40]. J. R. Thompson, L.A. Marcelino, and M. F. Polz, Heteroduplexes in mixed-template amplifications: formation, consequence and elimination by 'reconditioning PCR'.,Nucleic Acids Res.,30(9),2002,2083-8.

[41]. S. F. Altschul et al., Gapped BLAST and PSI-BLAST: a new generation of protein database search programs., Nucleic Acids Res.,25(17), 1997,3389-402.

[42]. T. Z. Desantis et al., Greengenes: Chimera-checked 16S rRNA gene database and workbenchcompatible in ARB, Appl. Environ. Microbiol.,72(72),2006,5069-5072.

[43]. J. D. Thompson, T. J. Gibson, F. Plewniak, F. Jeanmougin, and D. G. Higgins, The ClustalX windows interface: flexible strategies for multiple sequence alignment aided by quality analysis tools. Nucleic Acids Res, Nucleic Acids Res.,25(25), 1997,4876-82.

[44]. J. Felsenstein, PHYLIP (Phylogeny Inference Package) version 3.6. Distributed by the author. Department of Genome Sciences, University of Washington, Seattle.,2005.

[45]. P.D. Schloss and J. Handelsman, Introducing DOTUR, a computer program for defining operational taxonomic units and estimating species richness., Appl. Environ. Microbiol.,71(3), 2005,1501-1506.

[46]. B. JENNIFER and Hughes, Counting the Uncountable: Statistical Approaches to Estimating Microbial Diversity, Appl. Environ. Microbiol.,67(10),2001, 4399-4406.

[47]. M. K. Chelius and E. W. Triplett, The Diversity of Archaea and Bacteria in Association with the Roots of Zea mays L., Microb. Ecol., 41(3),2001,252-263.12

[48]. Simberloff D. 1978. Use of rarefaction and related methods in ecology. Biological Data in Water Pollution Assessment: Quantitative and Statistical Analyses (DicksonKL, Cairns JJ \& Livingston RJ, eds). American Society for Testing and Materials, West Conshohocken, 1978, 150-165. 
[49]. W. Liesack, H. Weyland, and E. Stackebrandt, "Potential risks of gene amplification by PCR as determined by 16S rDNA analysisof a mixed-culture of strict barophilic bacteria," Microb. Ecol.,21(1),1991,191-8.

[50]. J. R. Cole et al., The Ribosomal Database Project: improved alignments and new tools for rRNA analysis., Nucleic Acids Res.,37(Database issue),2009,141-145.

[51]. H. Nacke et al., Pyrosequencing-based assessment of bacterial community structure along different management types in German forest and grassland soils., Plos One,6(2),2011,e17000-e17000.

[52]. H. Nacke et al., Pyrosequencing-based assessment of bacterial community structure along different management types in German forest and grassland soils., Plos One,6(2),2011,e17000-e17000.

[53]. S. Shivaji et al., Bacterial diversity of soil in the vicinity of Pindari glacier, Himalayan mountain ranges, India, using culturable bacteria and soil 16S rRNA gene clones., Extrem. Life Extreme Cond.,15(1),2011,1-22.

[54]. Bärbel U. Foesel, Manfred Rohde, and Jörg Overmann, Blastocatella fastidiosa gen. nov., sp. nov., isolated from semiarid savanna soil -The first described species ofAcidobacteria subdivision 4" Syst. Appl. Microbiol., 36(2), 2013, 82-89.

[55]. P. Hugenholtz,B. M. Goebel, and N. R. Pace, Impact of culture-independent studies on the emerging phylogenetic view of bacterial diversity., J. Bacteriol., 180(18), 1998, 4765-74.

[56]. S. M. Barns, S. L. Takala, and C. R. Kuske, Wide distribution and diversity of members of the bacterial kingdom Acidobacterium in the environment., Appl. Environ. Microbiol., 65(4),1999,1731-1737.

[57]. P. H. Janssen, Identifying the dominant soilbacterial taxa in libraries of 16S rRNA and 16S rRNA genes., Appl. Environ. Microbiol.,72(3), 2006,1719-28.

[58]. V. Saultcherkas and Y. Steinberger, Soil microbial diversity in the vicinity of a Negev Desert shrub--Reaumuria negevensis., Microb. Ecol., 61(1),2011,64-81.

[59]. M. Ventura et al., Genomics of Actinobacteria: tracing the evolutionaryhistory of an ancient phylum., Microbiol. Mol. Biol. Rev. Mmbr, 71(3),2007, 495-548.

[60]. Hogan C.Michael, Bacteria Encyclopedia of Earth eds. Sidney Draggan and C.J.Cleveland, National Council for Science and the Environment, Washington DC,2010.

[61]. Angélique Chanal et al., The desert of Tataouine: an extreme environment that hosts a wide diversity of microorganisms and radiotolerant bacteria, Environ. Microbiol., 8(3),2006,514-525.

[62]. E. Prestel, S. Salamitou, and M. S. Dubow, An examination of the bacteriophages and bacteria of the Namib desert, J. Microbiol., 46(4),2008,364-372.

[63]. J. Wang, "Advance in studies of relationship between decreased yieldsof soybean resulted by repeated cultivation and changes in soil environment," Chin. J Soil Sci, 31(6),2000, 270-272.

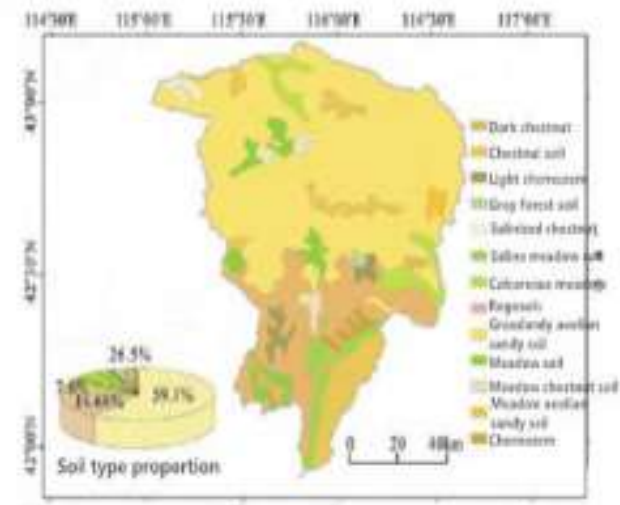

a

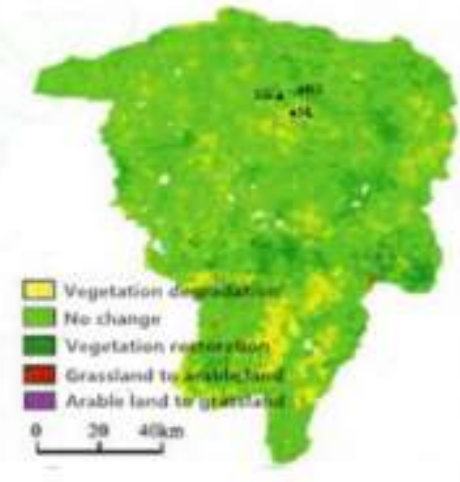

b

Fig. 1(a) The lacation and soil type of Plain Blue Banner (b) Vegetation coverage change in Plain Blue Banner during 2000-2009[14] and the collection sites of soil samples SL, SG and RS

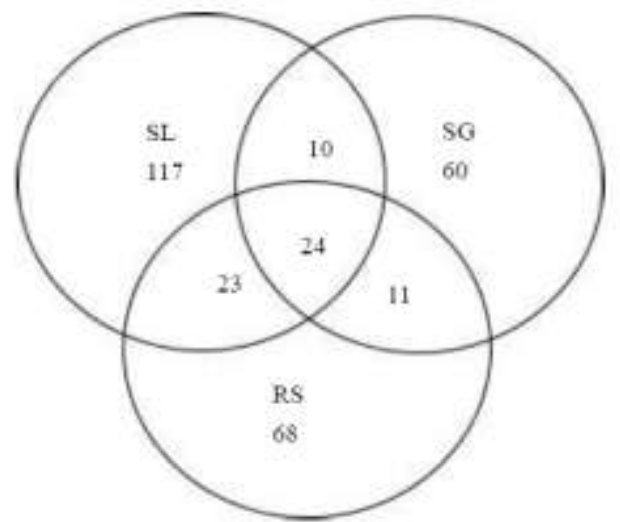

Fig. 2Venn diagram depicting OTUs from soil samples collected from sandy land (SL), sandy grassland (SG), and restored sandy grassland (RS) 


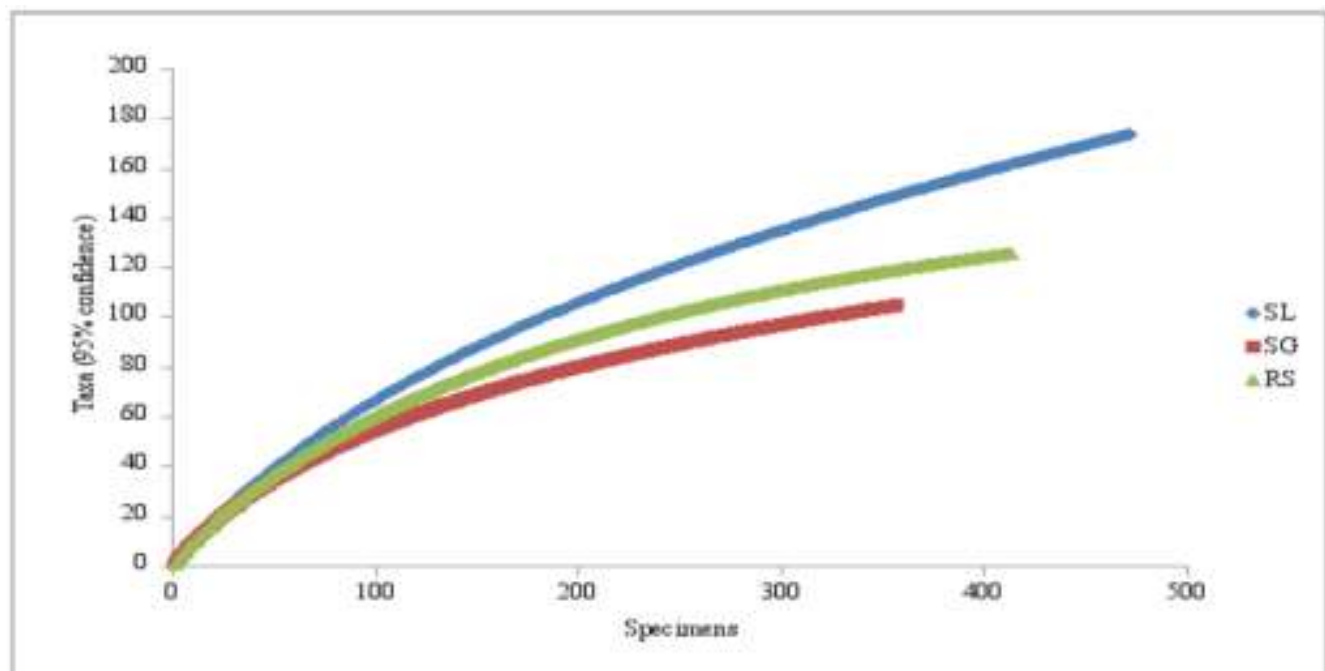

Fig. 3 Phylotype richness curves for clone and culture libraries. Sampling curves were calculated by rarefaction.

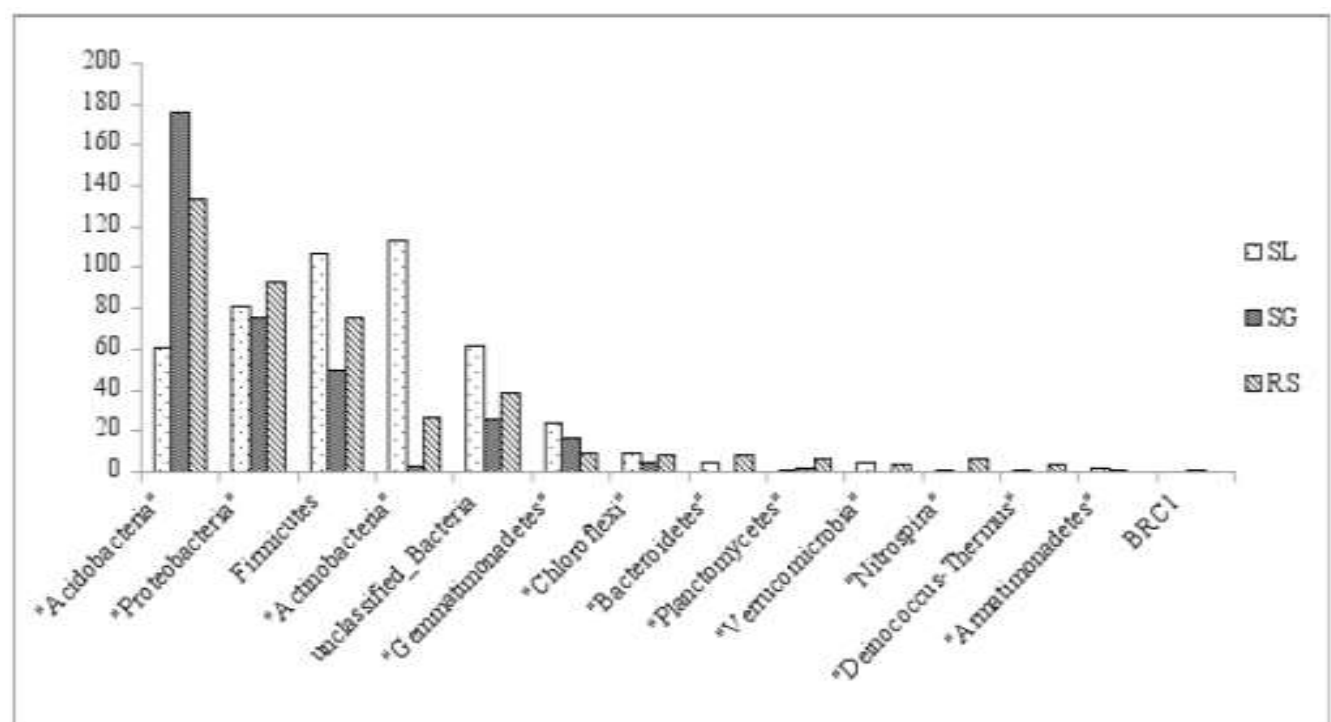

Fig. 4Phylum comparison of the bacterial community composition in soil samples collected from SL, SG, and $\mathrm{RS}$, as revealed by the $16 \mathrm{~S}$ rRNA gene.

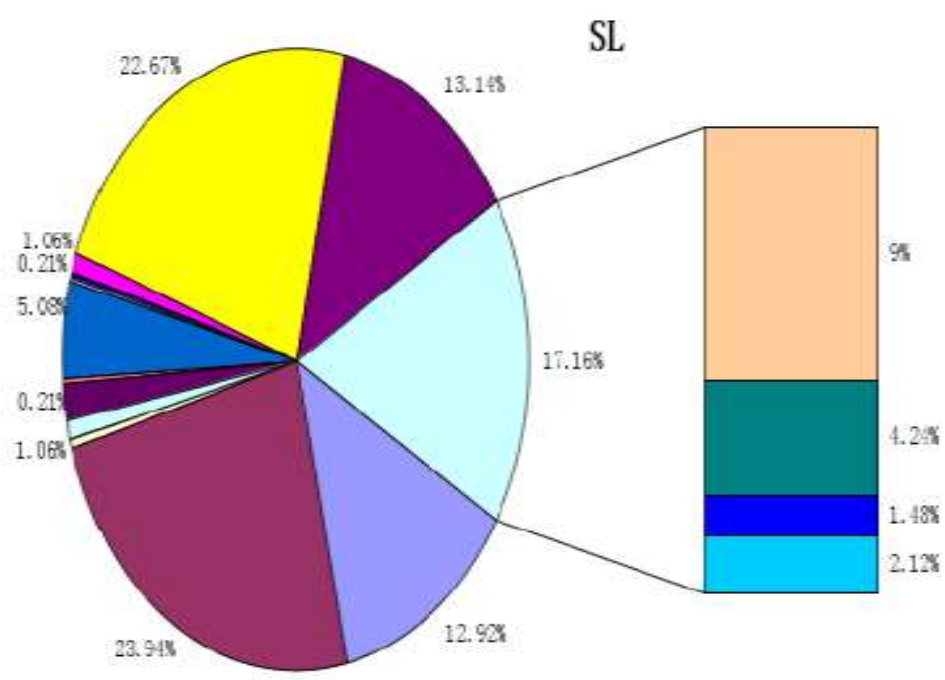

\begin{tabular}{|c|}
\hline D'Acidrbacteria' \\
\hline Q'Actindscteria' \\
\hline D'Anatinanaltes" \\
\hline D'Bact emidetes' \\
\hline "Chlordless' \\
\hline Deinscoccur-Therms' \\
\hline "Geanstionaletes' \\
\hline D'Xitropira' \\
\hline - 'Plsictongcetes' \\
\hline " 'Verruanicrobia" \\
\hline DFinictes \\
\hline $\mathrm{DBRCI}$ \\
\hline Wurclassified_Bateria \\
\hline DAlphapouteobacteria \\
\hline Batapreteobacteris \\
\hline Deltarnoteobacte \\
\hline
\end{tabular}



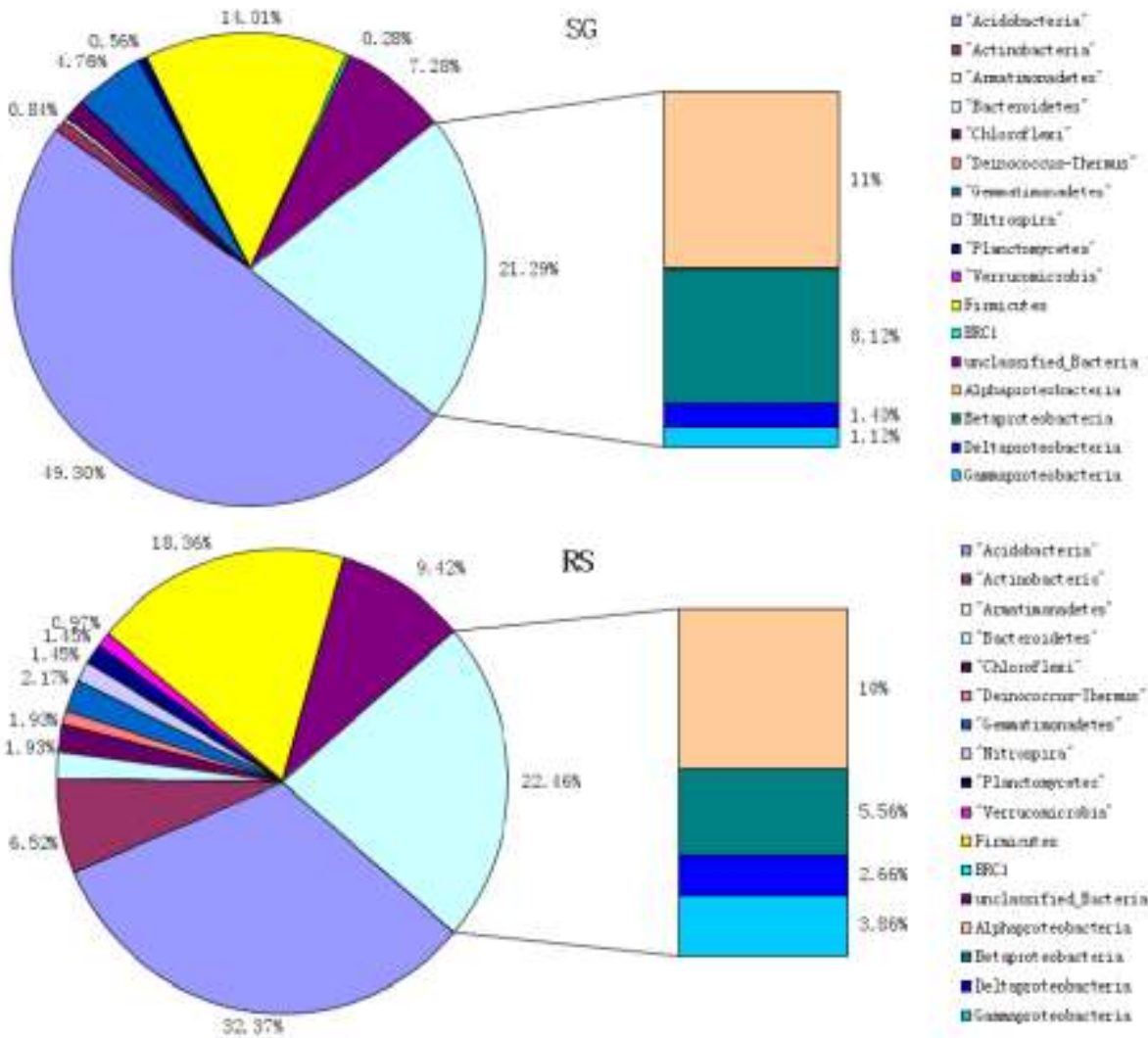

Fig. 5The percentage of each phylum and the classes (Alphaproteobacter, Betaproteobacter, Deltaproteobacter and Gammaproteobacter ) within the phylum Proteobacteria in the three sample sites (SL, SG and RS)

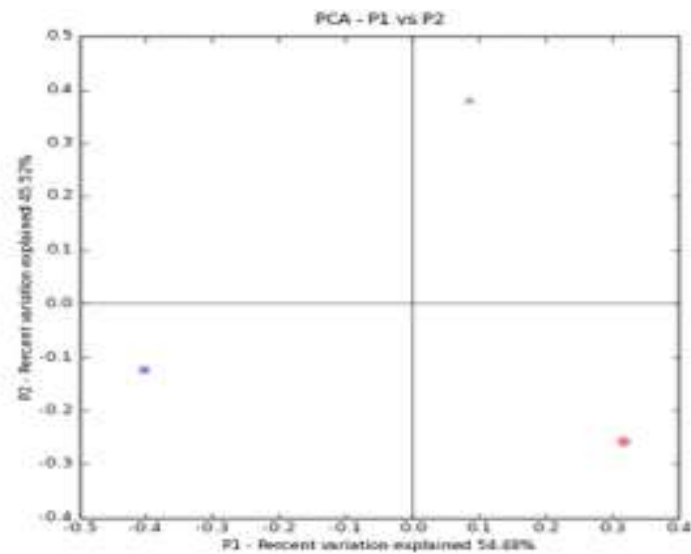

Raw P.Values: Psaiues have not been corrected for multiple comparisons

$\begin{array}{llcc}1 & 2 & 3 \\ 1 & 0.04000 .1500 \\ 2 & & 0.1700 \\ 3 & \end{array}$

Color Description
$(<0.001)$ Highly significant
$(0.001-0.01)$ Significant
$(0.01-0.05)$ Marginally significant
$(0.05-0.1)$ Suggestive
$(>0.1)$ Not significant

Fig. 6PCA plot based on environment properties ( $\square$ sandy land, $\bigcirc$ sandy grassland, $\triangle$ restored sandy grassland) of three soil samples (SL, SG, RS) and the unifrac significance of the three sample(1, SL; 2, SG; 3, RS).17 
Table 1.Statistical analyses of 16S rDNA clone libraries using standard ecological and molecular estimates of sequence diversity

\begin{tabular}{lccc}
\hline & SL & SG & RS \\
\hline Number of unique phylotypes & 117 & 60 & 68 \\
Taxa_S & 174 & 105 & 126 \\
Total number of clones & 472 & 359 & 414 \\
Dominance_D & 0.014 & 0.022 & 0.019 \\
Coverage (\%) & 80.297 & 87.640 & 89.614 \\
Menhinick & 8.009 & 5.565 & 6.193 \\
Evenness & 0.640 & 0.638 & 0.651 \\
Shannon_H & 4.713 & 4.205 & 4.406 \\
Simpson_1-D & 0.986 & 0.978 & 0.981 \\
Chao 1 Estimate & 335 & 162 & 156 \\
\hline
\end{tabular}

Table 2. Comparison of the three 16S rRNA gene libraries (SL, SG and RS) constructedusing three soil samples colledted from aeolian sandy land, sandy grassland, restored sandy land, based on BLAST analysis of the 16S rRNA gene sequences

\begin{tabular}{|c|c|c|c|c|c|c|}
\hline \multirow{2}{*}{ Phylum/ Class/ Order } & \multicolumn{2}{|c|}{ SL } & \multicolumn{2}{|c|}{ SG } & \multicolumn{2}{|c|}{ RS } \\
\hline & Number of clones & Library $(\%)$ & Number of clones & Library $(\%)$ & Number of clone & Library $(\%)$ \\
\hline Phylum- Acidobacteria & 61 & 12.9 & 176 & 49.3 & 134 & 33.4 \\
\hline Class-Acidobacteria & 61 & 12.9 & 176 & 49.3 & 134 & 33.4 \\
\hline Phylum- Actinobacteria & 113 & 23.9 & 3 & 0.8 & 27 & 6.5 \\
\hline Class-Actinobacteria & 113 & 23.9 & 3 & 0.8 & 27 & 6.5 \\
\hline Order-Acidimicrobiales & 31 & 6.6 & 0 & 0 & 3 & 0.7 \\
\hline Order-Actinomycetales & 46 & 9.7 & 1 & 0.3 & 18 & 4.3 \\
\hline Order-Solinbbrobacterales & 35 & 7.4 & 2 & 0.5 & 3 & 0.7 \\
\hline Order-Rubrobacterales & 0 & 0 & 0 & 0 & 3 & 0.7 \\
\hline Order-Thermoleophilales & 1 & 0.2 & 0 & 0 & 0 & 0 \\
\hline Phỵlum-Armatimonadetes & 2 & 0.4 & 1 & 0.3 & 0 & 0 \\
\hline Class-Armatimonadia & 2 & 0.4 & 1 & 0.3 & 0 & 0 \\
\hline Order-Armatimonadales & 2 & 0.4 & 1 & 0.3 & 0 & 0 \\
\hline Phylum- Bacteroidetes & 5 & 1.1 & 0 & 0 & 8 & 2.0 \\
\hline Class-Sphingobacteria & 5 & 1.1 & 0 & 0 & 8 & 2.0 \\
\hline Order-Sphingobacteriales & 5 & 1.1 & 0 & 0 & 8 & 2.0 \\
\hline Phylum-BRCl & 0 & 0 & 1 & 0.3 & 0 & 0 \\
\hline Phylum-Chloroflexi & 9 & 2.7 & 5 & 1.4 & 8 & 2.0 \\
\hline Class-Anaerolineae & 7 & 1.4 & 0 & 0 & 5 & 1.2 \\
\hline Order-Anaervlineales & 7 & 1.4 & 0 & 0 & 5 & 1.2 \\
\hline Class-Caldilineales & 5 & 1.1 & 0 & 0 & 0 & 0 \\
\hline Order-Caldilineales & 5 & 1.1 & 0 & 0 & 0 & 0 \\
\hline Class-Chloroflexi & 0 & 0 & 5 & 1.4 & 3 & 0.7 \\
\hline Order-Heipetosiphonales & 0 & 0 & 5 & 1.4 & 0 & 0 \\
\hline Order-Chloroflexales & 0 & 0 & 0 & 0 & 3 & 0.7 \\
\hline
\end{tabular}




\begin{tabular}{|c|c|c|c|c|c|c|}
\hline Order- Sphaerobacterales & 1 & 0.2 & 0 & 0 & 0 & 0 \\
\hline Phylum-Deinococcus-Thermus & 1 & 0.2 & 0 & 0 & 4 & 1.0 \\
\hline Class-Deinococci & 1 & 0.2 & 0 & 0 & 4 & 1.0 \\
\hline Order-Deinococcales & 1 & 0.2 & 0 & 0 & 4 & 1.0 \\
\hline Phylum- Firmicutes & 107 & 22.7 & 50 & 14.0 & 76 & 18.4 \\
\hline Class- Bacilli & 106 & 22.5 & 36 & 10.1 & 71 & 17.1 \\
\hline Order- Bacillales & 103 & 21.8 & 36 & 10.1 & 70 & 16.9 \\
\hline Order-Lactobacillales & 3 & 0.6 & 0 & 0 & 1 & 0.2 \\
\hline Class-Clostidia & 1 & 0.2 & 14 & 3.9 & 4 & 1.0 \\
\hline Order-Clostidiales & 1 & 0.2 & 14 & 3.9 & 4 & 1.0 \\
\hline Class-Negativicutes & 0 & 0 & 0 & 0 & 1 & 0.2 \\
\hline Order-Selenomonadales & 0 & 0 & 0 & 0 & 1 & 0.2 \\
\hline Phylum-Gemmatimonadetes & 24 & 5.1 & 17 & 4.7 & 9 & 2.2 \\
\hline Class-Gemmatimonadetes & 24 & 5.1 & 17 & 4.7 & 9 & 2.2 \\
\hline Order-Gemmatimonadales & 24 & 5.1 & 17 & 4.7 & 9 & 2.2 \\
\hline Phỵlum-Nitrospira & 1 & 0.2 & 0 & 0 & 6 & 1.4 \\
\hline Class-Nitrospira & 1 & 0.2 & 0 & 0 & 6 & 1.4 \\
\hline Order-Nitrospirales & 1 & 0.2 & 0 & 0 & 6 & 1.4 \\
\hline Phylum-Plactomycetes & 1 & 0.2 & 2 & 0.6 & 6 & 1.4 \\
\hline Class-Planctomycetacia & 1 & 0.2 & 2 & 0.6 & 3 & 0.7 \\
\hline Order-Planctomycetales & 1 & 0.2 & 2 & 0.6 & 3 & 0.7 \\
\hline Class-Phycisphaerae & 0 & 0 & 0 & 0 & 3 & 0.7 \\
\hline Order-Phycisphaerales & 0 & 0 & 0 & 0 & 3 & 0.7 \\
\hline Phylum- Proteobacteria & 81 & 17.2 & 76 & 21.2 & 93 & 22.5 \\
\hline Class-Alphaproteobacteria & 44 & 9.3 & 38 & 10.6 & 43 & 10.4 \\
\hline Order-Rhizobiales & 33 & 7.0 & 26 & 7.2 & 41 & 9.9 \\
\hline Order-Sphingomonadales & 10 & 2.1 & 1 & 0.3 & 1 & 0.2 \\
\hline Order-Caulobacterales & 1 & 0.2 & 0 & 0 & 0 & 0 \\
\hline Order-Rhodobacterales & 0 & 0 & 4 & 1.1 & 1 & 0.2 \\
\hline Order- Rhodospirillales & 0 & 0 & 7 & 2.0 & 0 & 0 \\
\hline Class- Betaproteobacteria & 20 & 4.2 & 29 & 8.1 & 23 & 5.6 \\
\hline Order-Burkholderiales & 17 & 3.6 & 20 & 5.6 & 13 & 3.1 \\
\hline Order- Hyarogenophilales & 1 & 0.2 & 0 & 0 & 0 & 0 \\
\hline Order-Rhodocyclales & 2 & 0.4 & 7 & 2.0 & 2 & 0.5 \\
\hline Order-Nitrosomonadales & 0 & 0 & 2 & 0.6 & 8 & 1.9 \\
\hline Class-Deltaproteobacteria & 7 & 1.5 & 5 & 1.4 & 11 & 2.7 \\
\hline Order-Myxococcales & 5 & 1.1 & 5 & 1.4 & 11 & 2.7 \\
\hline Order-Syntrophobacterales & 1 & 0.2 & 0 & 0 & 0 & 0 \\
\hline Order-Desulfuromonadales & 1 & 0.2 & 0 & 0 & 0 & 0 \\
\hline Class-Gammaproteobacteria & 10 & 2.1 & 4 & 1.1 & 16 & 3.9 \\
\hline Order-Legionellales & 3 & 0.6 & 0 & 0 & 7 & 1.7 \\
\hline Order-Xanthomonadales & 7 & 1.5 & 3 & 0.8 & 1 & 0.2 \\
\hline Order-Pseudomonadales & 0 & 0 & 0 & 0 & 8 & 1.9 \\
\hline
\end{tabular}




\begin{tabular}{lllllll} 
Order- Methlococcales & 0 & 0 & 1 & 0.3 & 0 & 0 \\
Phylum- Verrucomicrobia & 5 & 1.1 & 0 & 0 & 4 & 0.9 \\
Class- Opititae & 2 & 0.4 & 0 & 0 & 0 & 0 \\
$\quad$ Order- Opintales & 2 & 0.4 & 0 & 0 & 0 & 0 \\
Class- Spartobacteria & 3 & 0.6 & 0 & 0 & 0 & 0 \\
Class- Vernicomicrobiae & 0 & 0 & 0 & 0 & 2 & 0.5 \\
$\quad$ Order- Vemricomicrobiales & 0 & 0 & 0 & 0 & 2 & 0.5 \\
Unclassified-Bacteria & 62 & 13.1 & 26 & 7.2 & 39 & 9.4 \\
Total & $\mathbf{4 7 2}$ & 100 & 359 & 100 & 414 & 100 \\
\hline
\end{tabular}

The bold values represent the number of clones in each phylum and the number of clones in each library 